\title{
The Suggested Importance of PBRM1 Mutation in Predicting Postoperative Recurrence of Localized Clear Cell Renal Cell Carcinoma
}

\author{
Hanan Goldberg, MD, MSc \\ Urology Department, SUNY Upstate Medical University, Syracuse, NY
}

In the USA alone, cancers of the kidney and renal pelvis afflict over 70,000 individuals, causing approximately 14,000 deaths per year. ${ }^{1}$ Clear cell renal cell carcinoma (ccRCC) is the most prevalent histological subtype of kidney cancer and is a known immunogenic tumor. ${ }^{2}$ Although von Hippel-Lindau (VHL) is the initiating event in ccRCC, recent advances in next-generation sequencing (NGS) have led to the identification of additional genes that are frequently mutated in ccRCC, such as SETD2, KDM6A, $K D M 5 C, B A P 1$, and polybromo-1 (PBRM1), being found in approximately $40-50 \%$ of ccRCC. ${ }^{3-5}$ Although $70 \%$ of RCC will manifest as localized disease, approximately $33 \%$ will relapse following surgical removal. ${ }^{6}$ The probability of recurrence depends substantially on various clinical and histopathological features, which have been incorporated into approximately 20 different heterogeneous scoring systems. ${ }^{7-9}$

There are several scoring systems that utilize clinical and pathological data to predict recurrence in patients with ccRCC, including the UCLA integrated staging system, SSIGN, and Leibovich scores. Although very useful, these scoring systems are limited, which has lead to growing interest in the use of molecular biomarkers such as singlenucleotide polymorphism (SNP) signatures, showing promising results, ${ }^{10}$ and immunohistochemistry (IHC)based assays ${ }^{11}$ to improve prognostication. There are several genetic expression panels that have been used as

(C) Society of Surgical Oncology 2021

First Received: 27 November 2020

Accepted: 19 January 2021;

Published Online: 8 February 2021

H. Goldberg, MD, MSc

e-mail: gohanan@gmail.com biomarkers to differentiate ccRCC with high and low likelihood of recurrence. These include the ClearCode 34 , a 34-gene expression panel, ${ }^{12}$ and a 16-gene panel that was shown to be significantly associated with recurrence following stratification by stage, grade, and Leibovich score. $^{13}$

In the study published in this issue by Ohsugi et al., ${ }^{11}$ the authors developed a scoring system to predict recurrence based on clinicopathological factors incorporating PBRM1 expression. The authors retrospectively assessed 389 nonmetastatic ccRCC patients, with the primary endpoint of recurrence-free survival (RFS). A total of 53 patients $(13.6 \%)$ developed recurrence with median time of 61 months. Multivariable analyses showed that $\geq$ pT3, sarcomatoid or rhabdoid component, PBRM1 negativity, and necrosis were independent factors for RFS. The authors created a scoring system combined with these factors, naming it SSPN (Stage, Sarcomatoid, PBRM1 expression, and Necrosis) score. This score showed significant differences in RFS among various groups; low-risk group (no factors), intermediate-risk group (one factor), high-risk group (two or three factors), and very high-risk group (four factors). The authors also reported better predictive accuracy for 5-year-RFS with this new scoring system, with a higher area under the receiver operating characteristic curve than conventional risk models $(0.841$ versus $0.747-0.792$ ).

Lotan and Margulis ${ }^{14}$ recently pointed out that certain conditions must be met for the successful incorporation of new biomarkers, assisting in predicting recurrence in patients with localized renal cell carcinoma after nephrectomy. These include overcoming regulatory and financial obstacles and demonstrating a validated beneficial effect of adjuvant therapies, signifying the predictive and not only prognostic nature of these biomarkers. Future 
incorporation of these biomarkers will enable a personalized medicine approach rather than a one-size-fits-all model, which can result in over- or undertreatment.

Most ccRCC cases are associated with genetic alterations or epigenetic silencing of the von Hippel-Lindau (VHL) gene. This, in turn, results in an accumulation of hypoxia-inducible factors, driving dysregulated angiogenesis. ${ }^{15} \mathrm{CcRCC}$ has several secondary mutations, including Polybromo-1 (PBRM1) or BAF180, ${ }^{16}$ which is part of the switch/sucrose non-fermenting (SWI/SNF) chromatin remodeling complex. Like VHL, PBRMI maps to chromosome 3p, and PBRM1 mutation is the second most commonly mutated gene in ccRCC, present in approximately $40 \%$ of ccRCC. ${ }^{16}$ The PBRM1 protein is involved in various DNA repair mechanisms and is critical for cohesion between centromeres, necessary for maintaining genomic stability. ${ }^{17}$ PBRMI is a tumor suppressor gene, and this role is supported by in vitro experiments in ccRCC-derived cell lines, which show that PBRM1 gene silencing results in increased proliferation, migration, and colony formation. ${ }^{5}$ There is also data showing that loss of $P B R M 1$ in ccRCC dampens p53 function and especially p21 expression, which is key for cell cycle arrest and senescence. ${ }^{18}$ PBRM1 has also been shown to be a key regulator of tumor cell-autonomous immune response in RCC, with its loss of function likely contributing to the blunted immune checkpoint blockade response experienced by patients. ${ }^{19}$ In fact, the IMMOTION150 trial ${ }^{20}$ showed that tumors with high angiogenesis were enriched for PBRM1 mutations. Moreover, when comparing treatment outcomes in patients harboring tumors with PBRM1 mutations, there was a clear benefit in the progression-free survival of patients treated with a multitargeted receptor tyrosine kinase inhibitor (sunitinib) compared with patients treated with immune checkpoint inhibitors. These results suggest an association between angiogenesis and PBRMI mutations, indicating that $P B R M 1$-mutated patients may benefit more from antiangiogenics than from immunotherapy.

The scoring system created by Ohsugi et al. ${ }^{11}$ clearly incorporates important and influential risk factors predicting recurrence, in addition to presence of $P B R M 1$ mutation. The presence of sarcomatoid features, necrosis, and worsening stage has been shown to be individually associated with worse disease and higher recurrence rates. ${ }^{21}$ By incorporating the presence of $P B R M 1$ mutation with these known factors, the authors have succeeded in creating a scoring system with greater predictive ability than conventional risk models. The authors believe that this novel model may improve the prediction of oncologic outcomes of ccRCC and could facilitate shared clinical decisionmaking regarding whether to administer adjuvant therapy following radical surgery. Before wide clinical application of this scoring system can be recommended, validation would be required in larger prospective studies, with comparison with currently utilized conventional risk models.

DISCLOSURE Nothing to disclose. All authors report no conflicts of interest.

\section{REFERENCES}

1. Siegel RL, Miller KD, Jemal A (2019) Cancer statistics 2019. CA: Cancer J Clin. 2019; 69(1): 7-34.

2. Liu XD, Hoang A, Zhou L, et al. Resistance to antiangiogenic therapy is associated with an immunosuppressive tumor microenvironment in metastatic renal cell carcinoma. Cancer Immunol Res. 2015;3(9):1017-29.

3. Dalgliesh GL, Furge K, Greenman C, et al. Systematic sequencing of renal carcinoma reveals inactivation of histone modifying genes. Nature. 2010;463(7279):360-3.

4. Linehan WM, Ricketts CJ. The Cancer Genome Atlas of renal cell carcinoma: findings and clinical implications. Nat Rev Urol. 2019;16(9):539-52.

5. Varela I, Tarpey P, Raine K, et al. Exome sequencing identifies frequent mutation of the SWI/SNF complex gene PBRM1 in renal carcinoma. Nature. 2011;469(7331):539-42.

6. Janzen NK, Kim HL, Figlin RA, Belldegrun AS. Surveillance after radical or partial nephrectomy for localized renal cell carcinoma and management of recurrent disease. Urol Clin North Am. 2003;30(4):843-52.

7. Meskawi M, Sun M, Trinh QD, et al. A review of integrated staging systems for renal cell carcinoma. Eur Urol. 2012;62(2):303-14.

8. Patard JJ, Kim HL, Lam JS, et al. use of the University of California Los Angeles integrated staging system to predict survival in renal cell carcinoma: an international multicenter study. J Clin Oncol. 2004;22(16):3316-22.

9. Leibovich BC, Blute ML, Cheville JC, et al. Prediction of progression after radical nephrectomy for patients with clear cell renal cell carcinoma: a stratification tool for prospective clinical trials. Cancer. 2003;97(7):1663-71.

10. Wei JH, Feng ZH, Cao Y, et al. Predictive value of single-nucleotide polymorphism signature for recurrence in localised renal cell carcinoma: a retrospective analysis and multicentre validation study. Lancet Oncol. 2019;20(4):591-600.

11. Ohsugi H,Yoshida T, Ohe C, Ikeda J, Sugi M, Kinoshita H, Tsuta $\mathrm{K}$, Matsuda T. The SSPN score, a novel scoring system incorporating PBRM1 expression, predicts postoperative recurrence in patients with non-metastatic clear cell renal cell carcinoma. Ann Surg Oncol. 2020. https://doi.org/10.1245/s10434-020-09075-4.

12. Brooks SA, Brannon AR, Parker JS, et al. ClearCode34: A prognostic risk predictor for localized clear cell renal cell carcinoma. Eur Urol. 2014;66(1):77-84.

13. Rini B, Goddard A, Knezevic D, et al. A 16-gene assay to predict recurrence after surgery in localised renal cell carcinoma: development and validation studies. Lancet Oncol. 2015;16(6):676-85.

14. Lotan Y, Margulis V. Predicting recurrence in patients with localised renal cell carcinoma after nephrectomy. Lancet Oncol. 2019;20(4):473-5.

15. Liu XD, Yao J, Tripathi DN, et al. Autophagy mediates HIF2 $\alpha$ degradation and suppresses renal tumorigenesis. Oncogene. 2015;34(19):2450-60. 
16. Chad J, Creighton MM, Gunaratne PH, Wheeler DA, et al. Comprehensive molecular characterization of clear cell renal cell carcinoma. Nature. 2013;499(7456):43-9.

17. Brownlee PM, Chambers AL, Cloney R, Bianchi A, Downs JA. BAF180 promotes cohesion and prevents genome instability and aneuploidy. Cell Rep. 2014;6(6):973-81.

18. Cai W, Su L, Liao L, et al. PBRM1 acts as a p53 lysine-acetylation reader to suppress renal tumor growth. Nat Commun. 2019;10(1):5800.

19. Liu XD, Kong W, Peterson CB, et al. PBRM1 loss defines a nonimmunogenic tumor phenotype associated with checkpoint inhibitor resistance in renal carcinoma. Nat Commun. 2020;11(1):2135
20. McDermott DF, Huseni MA, Atkins MB, et al. Clinical activity and molecular correlates of response to atezolizumab alone or in combination with bevacizumab versus sunitinib in renal cell carcinoma. Nat Med. 2018;24(6):749-57.

21. Chin AI, Lam JS, Figlin RA, Belldegrun AS. Surveillance strategies for renal cell carcinoma patients following nephrectomy. Rev Urol. 2006;8(1):1-7.

Publisher's Note Springer Nature remains neutral with regard to jurisdictional claims in published maps and institutional affiliations. 